\title{
A Sensor based IoT Monitoring System for Electrical Devices using Blynk framework
}

\author{
Dr. P. Karuppusamy, \\ Professor, \\ Department of EEE, \\ Shree Venkateshwara Hi-Tech Engineering College, \\ Erode, India. \\ Email id: pkarrupusamyphd@gmail.com
}

\begin{abstract}
Electrical device monitoring is an essential work for improving the efficiency of electrical energy. The industrial device monitoring process are majorly contributed with physical verification of the process going on with the electrical instrument. In some cases the monitoring work is handled with help of automated sensor controllers. The automated sensor controllers are widely used for emergency cases of the ongoing process by the electrical instruments. The system status will be displayed on a screen when the system is fully controlled by an intelligent controller. From the status certain process and equipment are able to manage by physical switches by a human operator. The proposed work is designed to control certain applications which are remotely placed from the control station. The design utilizes internet medium and Blynk server for the specific operations. A sensor based monitoring station is kept near to the electrical device for sending the status of the application system. By using this design any system can be monitored remotely without physical verification. This improves the efficiency of energy utilization by the control devices.
\end{abstract}

Keywords: Remote monitor, IoT monitoring system, IoT control, Blynk control, Android control.

\section{Introduction}

Electrical appliances have become a part of every human's daily life. Certain electrical appliances like water heater, induction stove consumes more amount of electrical energy for its operation. Hence it is always must to monitor the running condition of certain applications. People may forget to switch off the appliances when they go out for longer hours. This may results in severe damage to the appliances as well as it increases the energy consumption costs. In order to avoid such conditions an IoT based monitoring and control system is introduced in the proposed work. The system is made to compatible with any number of appliances in the home and industry. The industrial applications are larger in their rating and consumes very high energy for its production and running purpose. During emergency time and maintenance time, the proposed system will be useful for the technician to control and verify a device by his own hand. This improves the reliability of maintenance operation and saves life of technician. The application also be useful for several remote monitoring applications. The basic components of IoT based remote applications is shown n figure 1.

The IoT control application needs a smart phone to act as an input/output device for reading the status of the connected electrical appliance or device. The smart phone is connected to the IoT hardware through wireless connection. The wireless connections are can be WiFi, Ethernet, Bluetooth or internet. The smartphone must contain a compatible operating system for communicating with the IoT hardware. Android OS is a most compatible OS for several IoT hardwares. There are several number of IoT hardwares are available in the market with several features. One can choose a better hardware for their specific application. The most popular IoT hardwares are Arduino, Raspberry Pi, NodeMCU, or ESP32.

ISSN: 2582-3825 (online) 
Journal of Electronics and Informatics (2020)

Vol.02/ No.03

Pages: 182-187

http://www.irojournals.com/iroei/

DOI: https://doi.org/10.36548/jei.2020.3.005

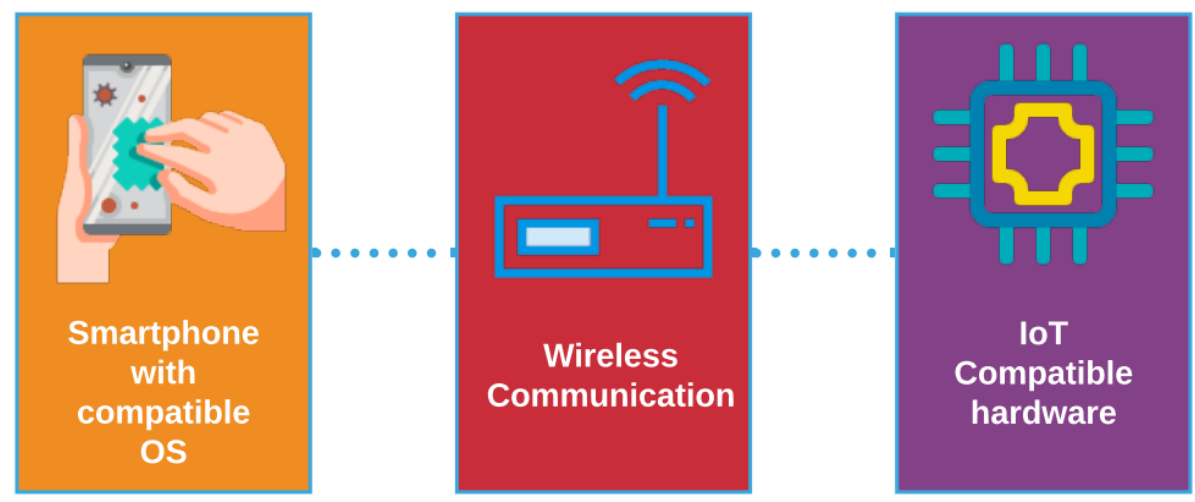

Figure 1. Components of IoT control system.

The proposed work is connected through Blynk IoT server for control applications. Here the status of the connected hardware pins are continuously transmitted to the Blynk server and the smartphone installed with Blynk application gets the ability to read the saved data from the IoT server. The hardware is also controllable from the smartphone through wireless connection with the help of Blynk hardware. In order to connect an IoT hardware with Blynk server, it is must to install the respective Blynk library to the IoT hardware unit. The architecture of the proposed IoT control system through Blynk hardware is shown in the figure 2.

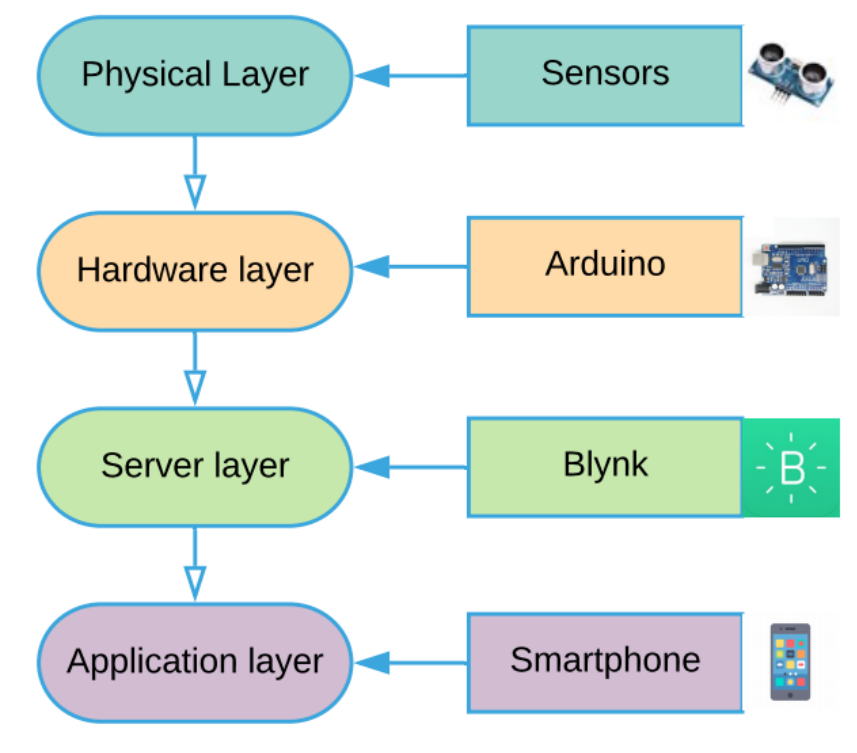

Figure 2. Architecture of the proposed IoT system.

\section{Related Work}

A home automations system based on Blynk application [1] was proposed to monitor the status of domestic appliances in the home. The work was designed with a solar powered house to utilized power from the sun light and it has a battery backup for saving the unused power generated by the solar panel. The design saves lot of power wasted by the unused domestic appliances in the home and it improves the efficiency of the power generated by the solar power station. The Blynk application is connected here with the hardware through WiFi protocol for communication.

ISSN: 2582-3825 (online) 
Journal of Electronics and Informatics (2020)

Vol.02/ No.03

Pages: 182-187

http://www.irojournals.com/iroei/

DOI: https://doi.org/10.36548/jei.2020.3.005

To improve the security feature of the IoT server lot of block chain methods are evolved [2]. It protects the informations which are shared through the IoT server to some specific destination. The IoT servers have large number of space for storing the informations from so many transmitters. The gathered informations are stored in a cloud storage for certain time or longer time for accessing the information in future. As so many people are interfering the cloud storage, there are possibility for the informations to be hacked by an expert. In order to avoid such drawbacks these block chain methods are introduced to protect the informations.

A plant monitoring and classification system using machine learning algorithms [3] was developed to classify the health status of a plant. Here the plants are monitored with the help of sensors to analyze the health status of the plant. The health status is analyzed through a moisture sensor fixed near to the root under the soil. The analyzed data is gathered by a microcontroller for transmitting it to the Blynk server for saving the data for future purpose. From the data saved in the Blynk server the user can able to understand the needs of a plant and the user can switch 'ON' the water pump connected to the plant from a remote area. The data saved in the Blynk application is also be retrievable at any time for several purposes and here the data are taken to classify the health status of a plant using machine learning algorithms. The huge amount of data gathered by the Blynk application gives ability to train and test the machine learning classifiers. But the energy consumption is one of the major drawbacks involved in IoT systems [4]. The energy utilized in perception layer and transport layers are usually minimum in nature but when it comes to processing and network layer it increases to certain extent of higher energy utilization. So that lot of green energy architectures are tried to involve in IoT applications. An IoT based energy management system for heating ventilation and air conditioning system [5] has been designed to monitor the status of the air conditioned room with several sensors. The data collected by the sensors are transmitted to the Blynk server for saving in the cloud storage. The data from the cloud storage is further taken in to mathematical account for calculating efficiency of the air conditioning system. The design work achieves nearly $0.9 \mathrm{KWhr}$ of energy from the connected device.

The only drawback in IoT server connected systems are regarding its privacy alone. The IoT data has the ability to be misused easily at many circumstance. A distributed block chain architecture [6] was framed to address this issue to overcome the theft ability of the IoT server data. The distributed block chain module meets the security issues along with improving information exchange process. An IoT based plant growth monitoring system [7] was developed to monitor the lighting condition of the plant fields. The plant fields are fitted with different numbers of LED lights and those lights are connected to a remote monitoring IoT application. The IoT application has the ability to read the condition of the plant field and it enables the user to switch 'ON' or 'OFF' the LED lights in the field with respect to the necessary lighting conditions. The plant growth and its conditions has been analyzed in the work by changing the light intensity. An automatic IoT environment greenhouse was designed [8] to monitor the plantation growth in the greenhouse environment. The design is implemented with Netuino $3 \mathrm{WiFi}$ unit for transferring the measured moisture, temperature and light in the green house field. This improves the maintenance quality of the greenhouse fields. The sensor data is also continuously monitored in this design and the data are transferred to a cloud database for analyzing the measured data. Thorough this the quality of the plant growth can be improved by changing the atmosphere of the green house artificially.

An IoT based health monitoring system [9] was proposed to monitor patient's health at some specific interval to forward the measured data the remote practitioner. The data are transmitted through multiple path like GSM, WiFi and BLE mobile application. This improves the reliability of the proposed work. The critical data values are designed to send as an alert to the practitioner. A big data analysis technique was discussed [10] to calculate the measured values from the sensors connected to a patient locating in distance place. Due to continuous monitoring of health data, the IoT models generate huge data to the cloud storage. The challenging part in analyzing the big data is extraction of useful information from the big data saved on cloud storage. So that in the work MAHOUT technique was utilized to filter the transmission data at the transmission side itself. The MAHOUT is trained to transmit only the emergency data to the cloud storage for processing the data. An infrastructure monitoring system based on IoT in smart cities [11] was developed to understand the traffic data on the roads from the moving vehicles. The data collected from the vehicle sensors are transmitted to the cloud storage through wireless receiver connected in the streets. The data are further taken from the cloud storage for analyzing the condition of the road traffic along with environmental and atmosphere conditions.

ISSN: 2582-3825 (online)

Submitted: 25.07.2020

Accepted: 20.08.2020

Published: 27.08.2020 
Journal of Electronics and Informatics (2020)

Vol.02/ No.03

Pages: 182-187

http://www.irojournals.com/iroei/

DOI: https://doi.org/10.36548/jei.2020.3.005

The assistance of IoT systems in medical imaging field [12] was reviewed to analyze the merits and limitations of IoT transmission in medical fields. The medical images like X-Ray and Scans are usually taken at some particular place and the analysis of certain difficult cases will be forwarded to some high operation labs for determining the patient's condition. During such situation the traditional e-mail system would not be helpful all the time due to its limitation on transmission size. The review work analyze the IoT transmission efficiency in transmitting the medical images through cloud storage environment. An arthritis monitoring system [13] was proposed to monitor the health status of the arthritis patients in real-time operation. The microcontroller connected in the system continuously monitors the condition and transmits the data to the remotely locate doctor when there is an emergency value read out by the sensors. The work has been implemented with wrapping algorithm for analyzing the seriousness of the sensor data. A multi-objective optimization technique [14] was generated to optimize the energy consumption of the cloud system when maintaining the big data. The amount of energy consumption is varied with respect to the amount of data analyzed and processed in the cloud storage. The security threat on the cloud storage is also addressed with the multiobjective optimization technique to ensure the network security of the cloud data. An energy monitoring technique based on IoT framework [15] was developed to save energy consumption on some specific locations by continuously monitoring the place though several sensors. A group of sensors are connected here to form a smart meter to measure the voltage and current changes of the circuit with their maximum load utilization and power factor calculations. The reading are further communicated through an ad-hoc network to a destination for analyzing and controlling purpose.

\section{Proposed Work}

From the literature it has been clearly noted that an IoT monitoring system should not send continuous data to the cloud platform for saving the energy. Hence the data observed from the sensors are always need to be verified by an algorithm for transmitting only the useful and necessary informations. The proposed work is designed only for user preference monitoring purpose only. Here the user can read the status of the connected system at any time in the work. It never send the status of the process to the user at any critical condition. So that it helps the system to be an energy and data efficient system. This limitation makes the proposed system should not to be connected at an emergency or severe monitoring system. The hardware setup of the proposed model is shown in the figure 3 .

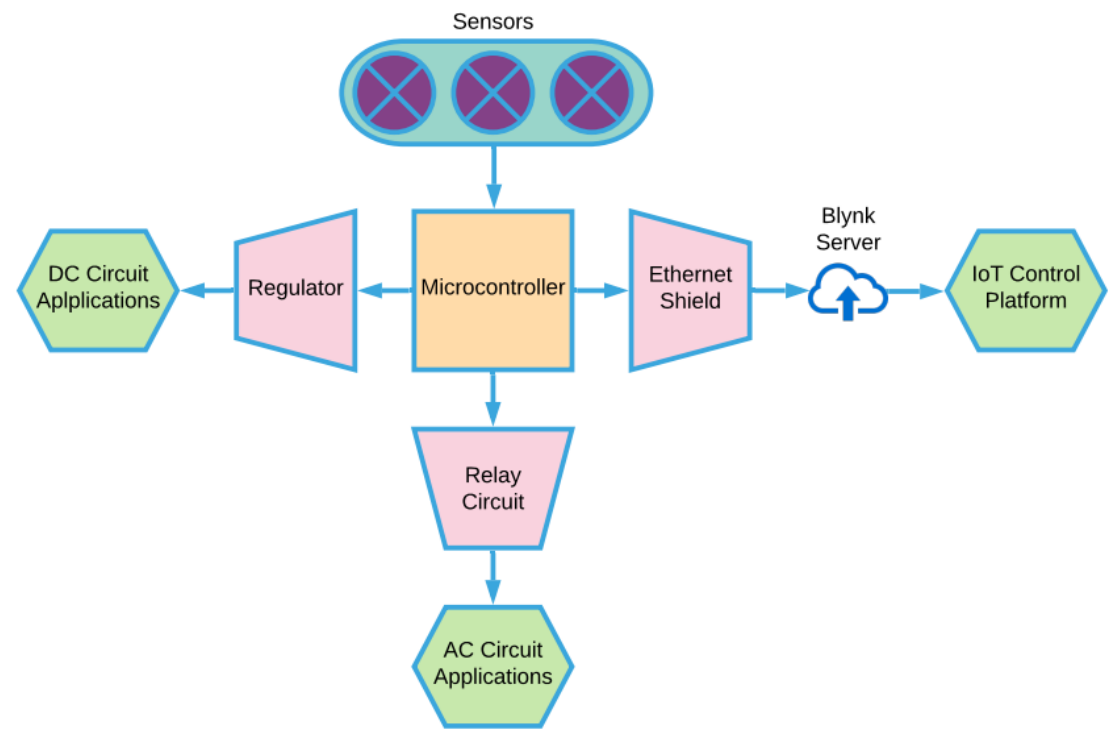

Figure 3. Hardware setup of the proposed IoT monitoring system.

ISSN: 2582-3825 (online)

Submitted: 25.07 .2020

Accepted: 20.08 .2020

Published: 27.08.2020 
Journal of Electronics and Informatics (2020)

Vol.02/ No.03

Pages: 182-187

http://www.irojournals.com/iroei/

DOI: https://doi.org/10.36548/jei.2020.3.005

The microcontroller unit of the proposed IoT monitoring system acts as a brain of the whole circuit. An arduino microcontroller is utilized in the work for connecting several peripheral units. The sensors are able to connect directly to the microcontroller without need of any other special components. The values taken from the sensors are utilized in the program written on the microcontroller to decide the operation status of the connected AC and DC circuits. The AC circuit connection of the circuit requires a simple relay circuit for enabling the switching operation. The proposed model is designed to do only switching operation to the applications. Hence there is no need of any other special control circuits for AC applications.

The DC applications are connected through a regulator circuit to enable the switching operations. Some the dc appliances working with higher voltage requires regulator circuit for increasing the voltage level signals produced by the microcontroller. A simple transistor switching circuit can also be enough in the proposed work for switching operations of the DC applications. A W5100 Ethernet shield is employed in the work to communicate with the IoT application from a remote place. The communication takes over through the Blynk server. Hence the library file of the Blynk has to be installed in the microcontroller software platform.

After installing the library file, the microcontroller has to be feed with the authentication number generated from the IoT application installed in an android device. For successful communication between the IoT application and the microcontroller, the authentication number has to be correctly entered in the program. The widget settings can be changed with respect to the design requirements of a circuit. The widget box of the Blynk application has so many types of display and input peripheral control blocks to be enabled by the user on his own preference. For normal switching operation a button switch available in the widget can be used and a simple LED display can be used for monitoring the switching state of the connected applications. The project creation process of the Blynk platform is shown in figure 4.

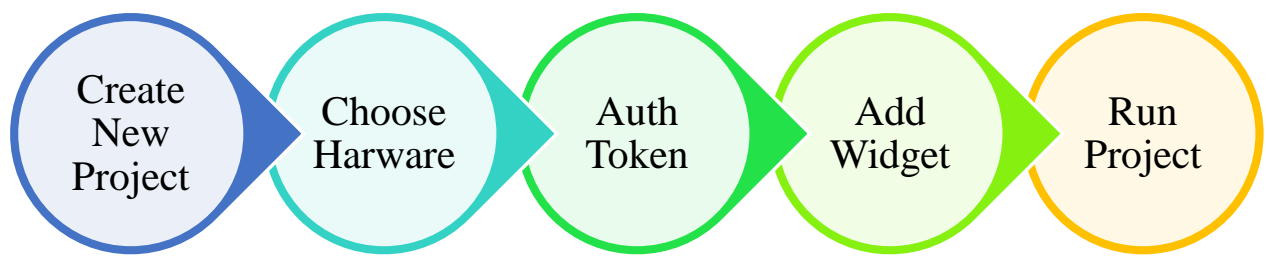

Figure 4. Blynk Platform creation process.

After creating the project an authentication code will be transferred to the registered user account from the Blynk server. That authentication code need to be pasted corrected before the setup loop of the program code as mentioned below and before that the hardware setup and shield which are employed in the work need to be chosen in the arduino project creation setup.

$$
\text { char auth }[]=\text { "ReceivedAuthCode" }
$$

\section{Results and Discussion}

In the proposed work the hardware system is connected several electrical appliances like lights, switching plugs of several heavy electrical appliances (heater, television, washing machine). The microcontroller has an in-built reset button to reset the switching operation controlled from the IoT server. Hence the system will gets the ability to control from both sides of the control operation. The multiple control option enables the connected hardware system as the error free system in the operation. The figure 5 indicates the switching operation status and monitoring status of the connected electrical devices in Blynk application.

ISSN: 2582-3825 (online) 


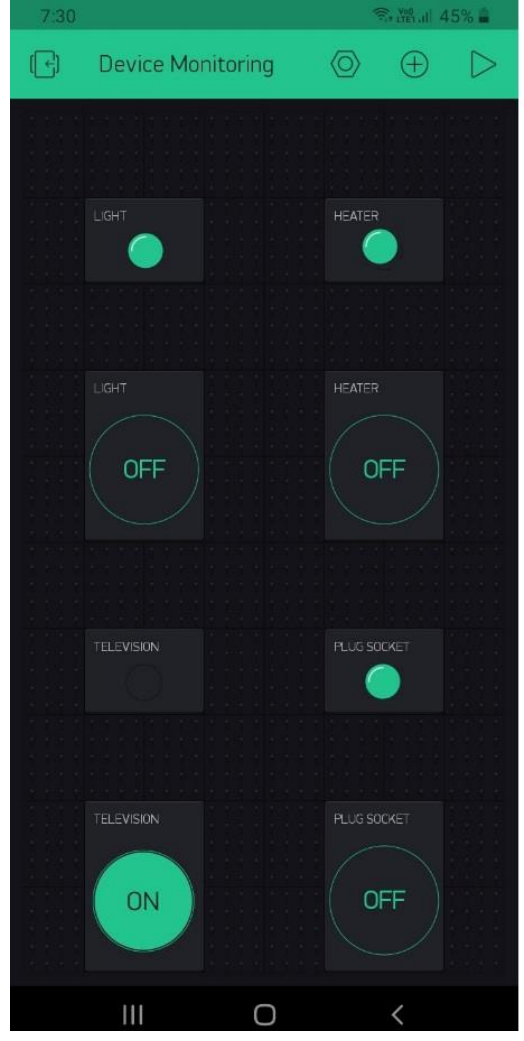

a

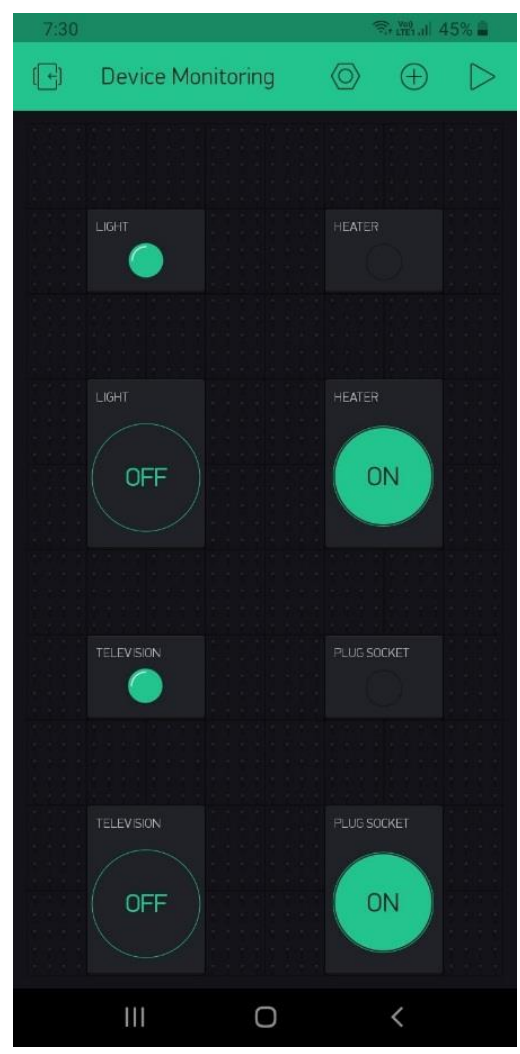

b

Figure 5. Experimental results of the proposed IoT monitoring system.

The proposed work has been verified with the Blynk framework. The project with title on device monitoring has been created in the framework. The hardware unit of the project is connected with a light unit, heater, television and a plug socket. The status of the appliances are verified through the relay units connected between the microcontroller and the appliances. The figure 5 (a) indicates that expect television all the other appliances are in 'ON' status. Hence the switch of the television shows ' $O N$ ' to enable the television circuit from the remote side. The status of the appliances are verified with several switching operations and few are shown in the figure 5(b) with modified status from the 5(a). From the observation of the verified circuit, the Blynk framework can be utilized for several controlling applications without any delay.

\section{Conclusion}

The efficiency of the electrical devices are must to be improved to save the energy consumption. As the electrical energy is one of most expensive source, it is always must to monitor the unused electrical appliances in the real life. Due to laziness and poor mindset, people used forget about the switching status of several appliances. The proposed IoT based monitoring system gives the ability to read the status of the connected electrical appliances from anywhere in the world and it also provides the control possibility to the user from the remote places. The proposed work is made with a reset switch to get a manual reset operation of the whole unit. The work utilizes the Blynk framework for the specific task and the results indicate that the motive of the proposed work is achieved in a prompt manner. 
Journal of Electronics and Informatics (2020)

Vol.02/ No.03

Pages: 182-187

http://www.irojournals.com/iroei/

DOI: https://doi.org/10.36548/jei.2020.3.005

\section{References}

1. Bhattarai, Dherendra, Amit Kumar Singh, Sanjeev Newpaney, and Pawan Pyakurel. "Design and Prototype Implementation of a Renewable Energy-Powered Home with Home Automation System Using Internet of Things (IoT)." In Advances in Greener Energy Technologies, pp. 489-504. Springer, Singapore, 2020.

2. Sivaganesan, D. "Block chain enabled internet of things." Journal of Information Technology 1, no. 01 (2019): $1-8$.

3. Shibani, Kumari, KS Sendhil Kumar, and G. Siva Shanmugam. "An Effective Approach for Plant Monitoring, Classification and Prediction Using IoT and Machine Learning." In Artificial Intelligence and Evolutionary Computations in Engineering Systems, pp. 143-154. Springer, Singapore, 2020.

4. Bashar, D. A. "Review on sustainable green Internet of Things and its application." J. Sustain. Wireless Syst. 1, no. 4 (2020): 256-264.

5. Dhanalakshmi, S., M. Poongothai, and Kaner Sharma. "IoT Based Indoor Air Quality and Smart Energy Management for HVAC System." Procedia Computer Science 171 (2020): 1800-1809.

6. Haoxiang, Wang. "Trust management of communication architectures of internet of things." Journal of trends in Computer Science and Smart technology (TCSST) 1, no. 02 (2019): 121-130.

7. Harun, Ahmad Nizar, Norliza Mohamed, Robiah Ahmad, and Nurul Najwa Ani. "Improved Internet of Things (IoT) monitoring system for growth optimization of Brassica chinensis." Computers and Electronics in Agriculture 164 (2019): 104836.

8. Raj, Jennifer S., and J. Vijitha Ananthi. "Automation using IoT in greenhouse environment." Journal of Information Technology 1, no. 01 (2019): 38-47.

9. Swaroop, K. Narendra, Kavitha Chandu, Ramesh Gorrepotu, and Subimal Deb. "A health monitoring system for vital signs using IoT." Internet of Things 5 (2019): 116-129.

10. Smys, S., and Jennifer S. Raj. "Internet of things and big data analytics for health care with cloud computing." J. Inf. Technol 1, no. 01 (2019): 9-18.

11. Lv, Zhihan, Bin Hu, and Haibin Lv. "Infrastructure monitoring and operation for smart cities based on IoT system." IEEE Transactions on Industrial Informatics 16, no. 3 (2019): 1957-1962.

12. Chandy, Abraham. "A review on iot based medical imaging technology for healthcare applications." Journal of Innovative Image Processing (JIIP) 1, no. 01 (2019): 51-60.

13. Parthasarathy, P., and S. Vivekanandan. "A typical IoT architecture-based regular monitoring of arthritis disease using time wrapping algorithm." International Journal of Computers and Applications 42, no. 3 (2020): 222-232.

14. Mugunthan, S. R. "Security and Privacy Preserving Of Sensor Data Localization Based On Internet of Things." Journal of ISMAC 1, no. 02 (2019): 81-92.

15. Al-Turjman, Fadi, Chadi Altrjman, Sadia Din, and Anand Paul. "Energy monitoring in IoT-based ad hoc networks: An overview." Computers \& Electrical Engineering 76 (2019): 133-142.

ISSN: 2582-3825 (online) 